\title{
A Branch-and-Bound Procedure to Minimize Total Tardiness on One Machine with Arbitrary Release Dates *
}

\author{
Philippe Baptiste, Jacques Carlier and Antoine Jouglet \\ Heudiasyc, UMR CNRS 6599, Université de Technologie de Compiègne, \\ BP 20529, 60205 Compiègne, France \\ \{philippe.baptiste, jacques.carlier, antoine.jouglet\}@hds.utc.fr
}

\begin{abstract}
In this paper, we present a Branch-and-Bound procedure to minimize total tardiness on one machine with arbitrary release dates. We introduce new lower bounds and we generalize some well-known dominance properties. Our procedure handles instances as large as 500 jobs although some 60 jobs instances remain open. Computational results show that the proposed approach outperforms the best known procedures.
\end{abstract}

Key words: Scheduling, Single Machine, Total Tardiness

*This work has been partially financed by ILOG S.A., under research contract ILOG/GRADIENT 201 (2000-2001). 


\section{Introduction, General Framework}

In this paper we consider the scheduling situation where $n$ jobs $J_{1}, \ldots, J_{n}$ have to be processed by a single machine and where the objective is to minimize total tardiness. Associated with each job $J_{i}$, are a processing time $p_{i}$, a due date $d_{i}$, and a release date $r_{i}$. All data are supposed to be positive integers. A job cannot start before its release date, preemption is not allowed, and only one job at a time can be scheduled on the machine. The tardiness of a job $J_{i}$ is defined as $T_{i}=\max \left(0, C_{i}-d_{i}\right)$, where $C_{i}$ is the completion time of $J_{i}$. The problem is to find a feasible schedule with minimum total tardiness $\sum T_{i}$. The problem, denoted as $1\left|r_{i}\right| \sum T_{i}$, is known to be NP-hard in the strong sense [15].

A lot of research has been carried on the problem with equal release dates $1 \| \sum T_{i}$. Powerful dominance rules have been introduced by Emmons [10]. Lawler [12] has proposed a dynamic programming algorithm that solves the problem in pseudo-polynomial time. This algorithm has been extended for scheduling serial batching machines by Baptiste and Jouglet [3]. Finally, $\mathrm{Du}$ and Leung have shown that the problem is NP-Hard [9]. Most of the exact methods for solving $1 \| \sum T_{i}$ strongly rely on Emmons' dominance rules. Potts and Van Wassenhove [14], Chang et al. [6] and Szwarc et al. [16], have developed Branch-and-Bound methods using the Emmons rules coupled with the decomposition rule of Lawler [12] together with some other elimination rules. The best results have been obtained by Szwarc, Della Croce and Grosso $[16,17]$ with a Branch-and-Bound method that efficiently handles instances with up to 500 jobs.

There are less results on the problem with arbitrary release dates. Chu 
and Portmann [7] have introduced a sufficient condition for local optimality which allows them to build a dominant subset of schedules. Chu [8] has also proposed a Branch-and-Bound method using some efficient dominance rules. This method handles instances with up to 30 jobs for the hardest instances and with up to 230 jobs for the easiest ones.

The aim of this paper is to propose an efficient Branch-and-Bound method with constraint propagation to solve the problem with arbitrary release dates. In the remaining parts of this introduction, we describe the general framework of our Branch-and-Bound procedure. The several "ingredients" that make it efficient will be described in the remaining sections.

In the following, a time window is associated with each job $J_{i}$; it represents the time interval within which each job can be scheduled. Before starting the search, the time window of $J_{i}$ is initially set to $\left[r_{i}, M\right)$, where $r_{i}$ is the release date of the job and where $M$ is a large value such as the largest release date plus the sum of the processing times. During the Branch-andBound, the time windows are tightened due to decisions taken at each node of the search tree together with constraint propagation and dominance rules. To keep it simple, we will say that the lower bound of the time window is the release date $r_{i}$ of $J_{i}$ (while this value can be larger than the initial release date). The upper bound of the time window $\delta_{i}$ will be referred to as the deadline of $J_{i}$.

In Constraint Programming terms, a variable $C_{i}$ representing the completion time of $J_{i}$ is associated with each job. Its domain is $\left[r_{i}+p_{i}, \delta_{i}\right)$. The criterion is an additional variable $\bar{T}$ that is constrained to be equal to $\sum_{i} \max \left(0, C_{i}-d_{i}\right)$. Arc-B-Consistency (see for instance [13]) is used to 
propagate this constraint. It ensures that when a schedule has been found, the value of $\bar{T}$ is actually the total tardiness of the schedule. To find an optimal solution, we solve successive variants of the decision problem. At each iteration, we try to improve the best known solution and thus, we add an additional constraint stating that $\bar{T}$ is lower than or equal to the best solution minus 1 . It now remains to show how to solve the decision variant of the problem.

We rely on the edge-finding branching scheme (see for instance [4]). Rather than searching for the starting times of jobs, we look for a sequence of jobs. This sequence is built both from the beginning and from the end of the schedule. Throughout the search tree, we dynamically maintain several sets of jobs that represent the current state of the schedule (see Figure 1).

- $P$ is the sequence of the jobs scheduled at the beginning,

- $Q$ is the sequence of the jobs scheduled at the end,

- $N S$ is the set of unscheduled jobs that have to be sequenced between $P$ and $Q$,

- $P F \subseteq N S$ (Possible First) is the set of jobs which can be scheduled immediately after $P$,

- and $P L \subseteq N S$ (Possible Last) is the set of jobs which can be scheduled immediately before $Q$.

At each node of the search tree, a job $J_{i}$ is chosen among those in $P F$ and it is scheduled immediately after $P$. Upon backtracking, this job is removed from $P F$. The heuristic used to select $J_{i}$ comes from $[7,8]$ : Among jobs 
of $P F$ with minimal release date, select the job that minimizes the function $\max \left(r_{i}+p_{i}, d_{i}\right)$. Of course, if $N S$ is empty then a solution has been found and we can iterate to the next decision problem. If $N S \neq \emptyset$ while $P F$ or $P L$ is empty then a backtrack occurs. Moreover, several propagation rules relying on jobs time-windows, are used to update and adjust these sets. These rules, known as edge-finding [5, 2], are also able to adjust the time-windows according to the machine constraint.

Due to our branching scheme, jobs are sequenced from left to right, so it may happen that at some node of the search tree, all jobs of $N S$ have the same release date (the completion time of the last job in $P$ ). In such a case, to improve the behavior of the Branch-and-Bound method, we apply the dynamic programming algorithm of Lawler [12] to optimally complete the schedule.

Such a Branch-and-Bound procedure is very easy to implement on top of a constraint-based scheduling system such as ILOG Scheduler. Unfortunately, it does not perform well since no specific technique has been used in the above formulation to solve the total tardiness problem. In the following sections, we introduce dominance properties (Section 2), lower-bounds (Section 3) and constraint propagation techniques (Section 4) to improve the behavior of the Branch-and-Bound method. Finally, our computational results are reported in Section 5. 


\section{Dominance Properties}

A dominance rule is a constraint that can be added to the initial problem without changing the value of the optimum, i.e., there is at least one optimal solution of the problem for which the dominance holds. Dominance rules can be of prime interest since they can be used to reduce the search space.

In this section, we present a generalization of Emmons dominance rules that are valid either if preemption is allowed or if jobs have identical processing times. We also propose some dominance rules relying on the sequence $P$. Finally, we introduce an Intelligent Backtracking scheme.

\section{$2.1 \quad$ Emmons Rules}

We recall Emmons Rules and generalize them to take into account release dates. Unfortunately, these rules are not valid if preemption is allowed (or, for some of them, if jobs have identical processing times). Nevertheless, we will see in Section 3 that these rules can be used in a pre-processing phase before computing a preemptive lower-bound of the problem.

\subsubsection{Initial Emmons Rules}

Emmons [10] has proposed a set of dominance rules for the special case where release dates are equal $\left(1 \| \sum T_{i}\right)$. These rules allow us to deduce some precedence relations between jobs. Following Emmons notation, $A_{i}$ and $B_{i}$ are the sets of jobs that have to be scheduled, according to the dominance rules, respectively after and before $J_{i}$. In the following, we say that $J_{i}$ precedes $J_{k}$ when there is an optimal schedule in which $J_{i}$ precedes 
$J_{k}$ and for which all previously mentioned dominance properties hold.

Emmons Rule 1. $\forall i, k(i \neq k)$, if $p_{i} \leq p_{k}$ and $d_{i} \leq \max \left(\sum_{J_{j} \in B_{k}} p_{j}+p_{k}, d_{k}\right)$ then $J_{i}$ precedes $J_{k}\left(J_{i} \in B_{k}\right.$ and $\left.J_{k} \in A_{i}\right)$.

Emmons Rule 2. $\forall i, k(i \neq k)$, if $p_{i} \leq p_{k}$ and $d_{i}>\max \left(\sum_{J_{j} \in B_{k}} p_{j}+p_{k}, d_{k}\right)$ and $d_{i}+p_{i} \geq \sum_{J_{j} \notin A_{k}} p_{j}$, then $J_{k}$ precedes $J_{i}$.

Emmons Rule 3. $\forall i, k(i \neq k)$, if $p_{i} \leq p_{k}$ and $d_{k} \geq \sum_{J_{j} \notin A_{i}} p_{j}$, then $J_{i}$ precedes $J_{k}$.

\subsubsection{Generalized Emmons Rules $\left(1\left|r_{i}, p m t n\right| \sum T_{i}\right)$}

Our aim is to generalize Emmons rules to the situation where we have arbitrary release dates. Such a generalization is relatively easy to do if we relax the non-preemption constraint. We will see that the resulting rules can be used to tighten the lower bound of the non preemptive problem (Section 3.4).

In the preemptive case, $J_{i}$ is said to precede $J_{k}$ if and only if $J_{k}$ starts after the end of $J_{i}$. As in Section 2.1.1, $A_{i}$ and $B_{i}$ respectively denote the set of jobs that are known to execute after and before $J_{i}$.

Note that active schedules are dominant, i.e., we only consider schedules in which jobs or pieces of jobs cannot be scheduled earlier without delaying another job. It is easy to see that all active schedules have exactly the same completion time $C_{\max }$. To compute this value, we can build the schedule where jobs are scheduled in non decreasing order of release dates. Now we can tighten the deadlines since each job $J_{i}$ cannot be completed after $C_{\max }-\sum_{J_{j} \in A_{i}} p_{j}$

In the following, we note $C_{\max }(E)$ the completion time of active schedules of a subset $E$ of jobs (other jobs are not considered). As mentioned 
previously, all active schedules have the same completion time and it can be computed in polynomial time. $C_{\max }(E)$ is a lower bound of the maximal completion time of the jobs of $E$ in any schedule of $\left\{J_{1}, \ldots, J_{n}\right\}$. If $B_{i}$, as in Section 2.1.1, is a set of jobs that have to be processed before $J_{i}$ then $J_{i}$ cannot start before $C_{\max }\left(B_{i}\right)$, i.e., the release date $r_{i}$ can be adjusted to $\max \left(r_{i}, C_{\max }\left(B_{i}\right)\right)$.

First, we make the following remark which allows us to compare the values of the tardiness of a job $J_{i}$ in two schedules $S$ and $S^{\prime}$.

Remark 1. Let $C_{i}$ and $C_{i}^{\prime}$ be the completion times of $J_{i}$ on two preemptive schedules $S$ and $S^{\prime}$ and let $T_{i}$ and $T_{i}^{\prime}$ be respectively the tardiness of $J_{i}$ in $S$ and $S^{\prime}$.

- If $C_{i}^{\prime}<C_{i}$ and $d_{i} \leq C_{i}$ then $T_{i}^{\prime}-T_{i}=\max \left(C_{i}^{\prime}, d_{i}\right)-C_{i} \leq 0$ (see Figure 2).

- If $d_{i} \geq \max \left(C_{i}, C_{i}^{\prime}\right)$ then $J_{i}$ is on time in $S$ and in $S^{\prime}$ and $T_{i}^{\prime}-T_{i}=0$.

Generalized Emmons Rule 1. Let $J_{i}$ and $J_{k}$ be two jobs such that $r_{i} \leq r_{k}$, $p_{i} \leq p_{k}$ and $d_{i} \leq \max \left(r_{k}+p_{k}, d_{k}\right)$, then $J_{i}$ precedes $J_{k}\left(J_{i} \in B_{k}\right.$ and $\left.J_{k} \in A_{i}\right)$.

Generalized Emmons Rule 2. Let $J_{i}$ and $J_{k}$ be two jobs such that $r_{i} \leq r_{k}$, $d_{i} \leq d_{k}$ and $\delta_{i} \leq d_{k}+p_{k}$, then $J_{i}$ precedes $J_{k}$

Generalized Emmons Rule 3. Let $J_{i}$ and $J_{k}$ be two jobs such that $r_{i} \leq r_{k}$ and $\delta_{i} \leq d_{k}$, then $J_{i}$ precedes $J_{k}$.

We now prove all these rules in the following single proof. 
Proof. Consider a schedule $S$ in which job $J_{i}$ and job $J_{k}$ satisfy the assumptions of one of the Generalized Emmons Rules. First, assume that job $J_{i}$ is completed before job $J_{k}$. Rescheduling the pieces of the two jobs, such that all pieces of $J_{i}$ are completed before the pieces of $J_{k}$, can only decrease the tardiness of $J_{i}$, and leave unchanged the tardiness of $J_{k}$.

Now, assume that job $J_{k}$ is completed before job $J_{i}\left(C_{k}<C_{i}\right)$. Let us reschedule the pieces of $J_{i}$ and $J_{k}$ such that all pieces of $J_{i}$ are completed before the pieces of $J_{k}$ (see Figure 3). Note that the exchange is valid since $r_{i}$ is lower than or equal to $r_{k}$. We show that this exchange does not increase total tardiness. Let $S^{\prime}$ be this new schedule and let $C_{i}^{\prime}$ be the completion time of $J_{i}$ in $S^{\prime}$. In $S^{\prime}, J_{k}$ is completed at time $C_{k}^{\prime}=C_{i}$. If $C_{i} \leq d_{i}$, the jobs $J_{i}$ and $J_{k}$ are on time in the two schedules $S$ and $S^{\prime}$ and the exchange has no effect. From now on, we suppose that $d_{i}<C_{i}$.

Since the completion times of all other jobs remain the same, the difference between the total tardiness of $S^{\prime}$ and of $S$ is exactly $\Delta=T_{i}^{\prime}-T_{i}+T_{k}^{\prime}-T_{k}$. Following Remark $1, T_{i}^{\prime}-T_{i}=\max \left(C_{i}^{\prime}, d_{i}\right)-C_{i}$ and $T_{k}^{\prime}-T_{k}=C_{k}^{\prime}-\max \left(C_{k}, d_{k}\right)=$ $C_{i}-\max \left(C_{k}, d_{k}\right)$.

Assume that $J_{i}$ and $J_{k}$ follow the assumptions of the Generalized Emmons Rule 1. We have, $\Delta=\max \left(C_{i}^{\prime}, d_{i}\right)-\max \left(C_{k}, d_{k}\right)$. Since $p_{i} \leq p_{k}$, note that all pieces of $J_{i}$ are completed before $C_{k}$ in $S^{\prime}$. We have then $C_{i}^{\prime} \leq C_{k}$ and $\Delta \leq \max \left(C_{k}, d_{i}\right)-\max \left(C_{k}, d_{k}\right)$. If $\max \left(r_{k}+p_{k}, d_{k}\right)=d_{k}$ then $d_{i} \leq d_{k}$, which leads to $\Delta \leq 0$. If $\max \left(r_{k}+p_{k}, d_{k}\right)=r_{k}+p_{k}$ then $d_{i} \leq r_{k}+p_{k} \leq C_{k}$ and $d_{k} \leq r_{k}+p_{k} \leq C_{k}$, which leads to $\Delta \leq C_{k}-C_{k}=0$.

Now, assume that $J_{i}$ and $J_{k}$ follow the assumptions of the Generalized Emmons Rule 2. We have $d_{k}+p_{k} \geq \delta_{i} \geq C_{i}$, thus $d_{k} \geq C_{i}-p_{k}$. All pieces 
of $J_{k}$ are scheduled between $C_{i}^{\prime}$ and $C_{i}$ so $C_{i}^{\prime} \leq C_{i}-p_{k}$ and $T_{i}^{\prime}-T_{i}=$ $\max \left(C_{i}^{\prime}, d_{i}\right)-C_{i} \leq \max \left(C_{i}-p_{k}, d_{i}\right)-C_{i}$. Thus $\Delta=T_{i}^{\prime}-T_{i}+T_{k}^{\prime}-T_{k} \leq$ $\max \left(C_{i}-p_{k}, d_{i}\right)-\max \left(C_{k}, d_{k}\right)$. We have $d_{i} \leq d_{k}$ and $C_{i}-p_{k} \leq d_{k}$ then $\Delta \leq 0$.

Now, assume that $J_{i}$ and $J_{k}$ follow the assumptions of the Generalized Emmons Rule 3. Note that we have $C_{i} \leq \delta_{i}$, which leads to $T_{i}^{\prime}-T_{i}=$ $\max \left(C_{i}^{\prime}, d_{i}\right)-C_{i} \leq 0$. We have $C_{k} \leq C_{i} \leq \delta_{i} \leq d_{k}$ then $J_{k}$ is on time in $S$ and in $S^{\prime}$. Thus $T_{k}^{\prime}-T_{k}=0$ and $\Delta \leq 0$.

In all cases, the exchange does not increase total tardiness.

As shown in Figure 4, the Generalized Emmons Rule 1, does not hold in the non-preemptive case. Indeed, $J_{1}$ would have to be completed before $J_{2}$, which is not true in the non-preemptive case.

\subsubsection{Applying Generalized Emmons Rules}

We can use these dominance rules one after the other to adjust the data of an instance: if a set $B_{i}$ of jobs is proved to precede $J_{i}$ according to the Generalized Emmons Rules, it is possible to adjust $r_{i}$ to $r_{i}=\max \left(r_{i}, C_{\max }\left(B_{i}\right)\right)$. Each rule can be implemented in $O\left(n^{2}\right)$. The jobs are sorted in non-decreasing order of release dates in a heap structure, which runs in $O(n \log n)$. For each job $J_{i}$, we consider the set of jobs which are completed before $J_{i}$ according to the Generalized Emmons Rules. Computing the earliest completion time of this set and adjusting $r_{i}$ runs in $O(n)$, since the jobs are already sorted in non-decreasing order of release dates. The heap structure is maintained in $O(\log n)$. Therefore, we have an overall time complexity of $O(n)$ for each job. New precedence dominances can be found and the rules might have to 
be applied again. Each job can be adjusted at most $n-1$ times. Hence, in the worst case the whole propagation is in $O\left(n^{3}\right)$.

\subsubsection{Equal Length Jobs}

We come back to the initial non-preemptive problem with release dates. We prove that, considering two jobs which have the same processing time, the first Emmons Rule is valid. Note that, contrary to the Generalized Emmons Rules, this dominance property is valid even in the non-preemptive case.

Theorem 1. Let $J_{i}$ and $J_{k}$ be two jobs such that $p_{i}=p_{k}$. If $r_{i} \leq r_{k}$ and $d_{i} \leq \max \left(r_{k}+p_{k}, d_{k}\right)$, then there exists an optimal schedule in which $J_{i}$ precedes $J_{k}$.

Proof. Consider a schedule $S$ in which job $J_{i}$ and job $J_{k}$ satisfy the assumptions and in which job $J_{k}$ is completed before job $J_{i}$. Let us exchange $J_{i}$ and $J_{k}$ in $S$. Note that the exchange is valid since $r_{i}$ is lower than or equal to the earliest start time of $J_{k}$. We have $p_{i}=p_{k}$, then the completion time of the other jobs do not change. This exchange does not increase total tardiness as it has been proved for the Generalized Emmons Rule 1. 


\subsection{Removing Dominated Sequences}

Several dominance properties have been introduced in $[7,8]$. These rules focus on the jobs in $N S$ plus the last job of $P$. They determine that some precedence constraints can be added. Such constraints allow us to adjust release dates and to filter $P F$. All these rules are used in our Branch-andBound procedure. On top of this, we also consider dominance properties that take into account the complete sequence $P$. Informally speaking, our most basic rule states that if the current sequence $P$ can be "improved", then it is dominated and we can backtrack.

In the following, let $C_{\max }(P)$ and $T(P)$ denote the completion time and the total tardiness associated with the current sequence $P$. Now consider a permutation $P^{\prime}$ of $P$ of and let us examine under which condition $P^{\prime}$ is "as good as" $P$.

- If $C_{\max }(P) \leq C_{\max }\left(P^{\prime}\right)$ and $T(P) \leq T\left(P^{\prime}\right)$, then we can replace sequence $P^{\prime}$ by sequence $P$ in any feasible schedule so sequence $P$ is at least "as good as" sequence $P^{\prime}$.

- Now, assume that we have $C_{\max }(P)>C_{\max }\left(P^{\prime}\right)$. Let $r_{\min }$ be the smallest release date of job belonging to set $N S$. If we replace sequence $P^{\prime}$ by sequence $P$ in a feasible schedule, all jobs in $N S \cup Q$ have to be shifted of at most: $\max \left(C_{\max }(P), r_{\min }\right)-\max \left(C_{\max }\left(P^{\prime}\right), r_{\min }\right)$ time units. So, the additional cost for jobs in $N S \cup Q$ is at most $(|N S|+|Q|)\left(\max \left(C_{\max }(P), r_{\min }\right)-\max \left(C_{\max }\left(P^{\prime}\right), r_{\min }\right)\right)$. Hence, $P$ is at least as good as $P^{\prime}$ if $T(P)+(|N S|+|Q|)\left(\max \left(C_{\max }(P), r_{\min }\right)-\right.$ $\left.\max \left(C_{\max }\left(P^{\prime}\right), r_{\min }\right)\right) \leq T\left(P^{\prime}\right)$. 
Now we can define the notion of "better" sequence:

Definition 1. We say that sequence $P$ is "better" than sequence $P^{\prime}$ if sequence $P$ is at least "as good as" sequence $P^{\prime}$ and if either (1) sequence $P^{\prime}$ is not at least "as good as" sequence P (2) or if sequence $P$ is lexicographically smaller than $P^{\prime}$.

To compare two sequences, we just have to build the schedules associated with them and this can be done in linear time.

We propose simple techniques to detect that a job $J_{i} \in P F$ cannot be actually scheduled just after $P$ and thus can be removed from $P F$. We note $P \mid J_{i}$ the sequence where $J_{i}$ is scheduled immediately after $P$. Informally speaking, our most basic rule states that if the sequence $P \mid J_{i}$ can be "improved", then it is dominated, so $J_{i}$ can be removed from set $P F$.

Theorem 2. Let $J_{i}$ be a job belonging to PF. If there exists a permutation $\pi$ which is "better" than $P \mid J_{i}$, then sequence $P \mid J_{i}$ is dominated.

Proof. If sequence $\pi$ is "better" than $P \mid J_{i}$, then we can replace sequence $P \mid J_{i}$ by sequence $\pi$ in any feasible schedule so $P \mid J_{i}$ is dominated.

If scheduling $J_{i}$ after $P$ leads to a dominated schedule, then $J_{i}$ can be removed from the set $P F$.

To find better permutations $\pi$ of $P \mid J_{i}$, we can enumerate all permutations $\pi$ that are identical to $P \mid J_{i}$ except for the $k$ last jobs. When $k$ is large, we have a great reduction of the search space but this takes a lot of time. Experimentally, $k=6$ seems to be a good trade-off between the reduction of the search tree and the time spent in the enumeration. 
We can also enumerate the permutations $\pi$ that are obtained from $P \mid J_{i}$ by inserting $J_{i}$ somewhere inside $P$ or by interchanging $J_{i}$ with another job of $P$. Since there are $O(n)$ such permutations and since comparing two sequences can be done in linear time, the algorithm runs in $O\left(n^{2}\right)$ for a given job $J_{i}$.

\subsection{Intelligent Backtracking}

Intelligent Backtracking techniques "record", throughout the search tree, some situations that do not lead to a feasible (non-dominated) solution.

Given a node $\nu$ of the search tree, let $P^{\nu}$ be the "value" taken by sequence $P$ at node $\nu$. Whenever a backtrack occurs, the node $\nu$ is stored in a "nogood" list. We only save: (1) the set of jobs belonging to sequence $P^{\nu},(2)$ the completion time $C_{\max }\left(P^{\nu}\right)$ of sequence $P^{\nu}$, and (3) the cost $T\left(P^{\nu}\right)$ associated with sequence $P^{\nu}$.

Now assume that we encounter another node $\mu$ such that sequence $P^{\nu}$ is a permutation of sequence $P^{\mu}$, i.e., the set of jobs belonging to sequence $P^{\mu}$ is exactly the set of jobs belonging to sequence $P^{\nu}$. If sequence $P^{\nu}$ is a "better" sequence (see Definition 1) than sequence $P^{\mu}$, then $\mu$ is dominated by $\nu$.

We use this information in two ways. First, we can use it to filter the set of possible first jobs $P F$. Suppose that scheduling a job $J_{i}$ just after the current sequence $P$ leads to a node $\mu$ which is dominated by a node $\nu$ which has been stored in the "no-good" list. The sequence $P \mid J_{i}$ is then dominated and we can remove $J_{i}$ from set $P F$. We can also use this information in the following way. When a backtrack occurs, the backtracking strategy can lead to a node $\mu$ which is dominated by a node $\nu$ which has been stored 
in the "no-good" list. We have then to backtrack again and that until the backtracking strategy could lead to a node which is not dominated.

With a view to using efficiently the "no-good" list, we use an HashTable. Most of the time, it allows us to obtain a pertinent state of the list in $O(n \log n)$. A state of the list consists of a set of jobs sorted in Lexicographic order coded with a list of pointers, and a list of couples $\left(T\left(P^{\kappa}\right), C_{\max }\left(P^{\kappa}\right)\right)$, also coded with a list of pointers, which fit to the different states of nodes $\kappa$ which have ever been visited with this set of jobs. Assume that we encounter a node $\mu$ such that the set of jobs belonging to sequence $P^{\mu}$ is the same than a state of the "no-good" list. Sorting jobs in Lexicographic order runs in $O(n \log n)$. Computing an index in the Hash-Table of this set of jobs runs in $O(n)$. Since jobs are sorted in Lexicographic order, comparing two sets of jobs runs in $O(n)$. If an associated couple $\left(T\left(P^{\kappa}\right), C_{\max }\left(P^{\kappa}\right)\right)$ of this state is such that its tardiness $T\left(P^{\kappa}\right)$ and its completion time $C_{\max }\left(P^{\kappa}\right)$ are respectively greater than or equal to those of sequence $P^{\mu}$, then this couple is replaced by the tardiness $T\left(P^{\mu}\right)$ and the completion time $C_{\max }\left(P^{\mu}\right)$ of sequence $P^{\mu}$.

\subsection{Adjusting Deadlines}

Consider a job $J_{i} \in N S$ and assume that the current node of the search tree can be extended to a feasible optimal schedule where $J_{i}$ is completed at some time point $C_{i}$. Let $P|\lambda| J_{i}|\mu| Q$ be the sequence of the jobs in the feasible optimal schedule and let us modify this sequence by removing $J_{i}$ and inserting it somewhere in $P$. The new sequence is $P^{\prime}\left|J_{i}\right| P^{\prime \prime}|\lambda| \mu \mid Q$, where $P^{\prime}\left|J_{i}\right| P^{\prime \prime}$ is the sequence derived from $P$ after the insertion of $J_{i}$. 
In the following, we assume that $r_{i} \leq C_{\max }\left(P^{\prime}\right)$. Under this hypothesis, it is easy to see that $C_{\max }\left(P^{\prime}\left|J_{i}\right| P^{\prime \prime} \mid \lambda\right) \leq C_{\max }\left(P|\lambda| J_{i}\right)$ and thus the tardiness of the jobs in $\boldsymbol{\mu} \mid \boldsymbol{Q}$ has not been increased. On top of that, the jobs in $\lambda$ have been shifted of at most $C_{\max }\left(P^{\prime}\left|J_{i}\right| P^{\prime \prime}\right)-C_{\max }(P)$ time units. Thus, the total tardiness of the jobs in $\boldsymbol{\lambda}$ has been increased of at most $|\lambda|\left(C_{\max }\left(P^{\prime}\left|J_{i}\right| P^{\prime \prime}\right)-C_{\max }(P)\right) \leq(|N S|-1)\left(C_{\max }\left(P^{\prime}\left|J_{i}\right| P^{\prime \prime}\right)-C_{\max }(P)\right)$. Finally, the total tardiness of the jobs in $\boldsymbol{P} \cup\left\{\boldsymbol{J}_{\boldsymbol{i}}\right\}$ has been increased of $T\left(P^{\prime}\left|J_{i}\right| P^{\prime \prime}\right)-\left(T(P)+\max \left(0, C_{i}-d_{i}\right)\right)$. Consequently, the total tardiness has been increased of at most

$(|N S|-1)\left(C_{\max }\left(P^{\prime}\left|J_{i}\right| P^{\prime \prime}\right)-C_{\max }(P)\right)+T\left(P^{\prime}\left|J_{i}\right| P^{\prime \prime}\right)-T(P)-\max \left(0, C_{i}-d_{i}\right)$.

Since the initial schedule is optimal, the above expression is greater than or equal to 0 and thus,

$$
C_{i} \leq d_{i}+(|N S|-1)\left(C_{\max }\left(P^{\prime}\left|J_{i}\right| P^{\prime \prime}\right)-C_{\max }(P)\right)+T\left(P^{\prime}\left|J_{i}\right| P^{\prime \prime}\right)-T(P) .
$$

In other words, the deadline $\delta_{i}$ of $J_{i}$ can be adjusted to

$$
\min \left(\delta_{i}, d_{i}+(|N S|-1)\left(C_{\max }\left(P^{\prime}\left|J_{i}\right| P^{\prime \prime}\right)-C_{\max }(P)\right)+T\left(P^{\prime}\left|J_{i}\right| P^{\prime \prime}\right)-T(P)\right)
$$

Since there are $O(n)$ sequences $P^{\prime}\left|J_{i}\right| P^{\prime \prime}$ (with $r_{i} \leq C_{\max }\left(P^{\prime}\right)$ ) that can be derived from $P$ and since the completion time and the total tardiness of a sequence can be computed in linear time, all adjustments of deadlines related to $J_{i}$ can be computed in $O\left(n^{2}\right)$.

\section{Lower Bounds}

Relaxing non-preemption is a standard technique to obtain lower bounds for non-preemptive scheduling problems. Unfortunately, the problem with no 
release dates (for which the preemption is not useful) is already NP-Hard, so some additional constraints have to be relaxed to obtain a lower bound in polynomial time. From now on, assume that jobs are sorted in nondecreasing order of due dates. First, Chu's lower bound is recalled then, a new lower bound is described and finally, we show how the Generalized Emmons Rules can be used to improve this lower bound.

\subsection{Chu's Lower Bound}

Chu [8] has introduced an $O(n \log n)$ lower bound, $\boldsymbol{l b}_{\boldsymbol{C h u}}$, that can be computed as follows. Preemption is relaxed and jobs are scheduled according to the SRPT (Shortest Remaining Processing Time) rule. Each time a job becomes available or is completed, a job with the shortest remaining processing time among the available and uncompleted jobs is scheduled (see Table 1 and Figure 5). The computation of the lower bound is based on Proposition 1 $([8])$.

\begin{tabular}{|l|l|l|l|}
\hline$J_{i}$ & $r_{i}$ & $p_{i}$ & $d_{i}$ \\
\hline \hline$J_{1}$ & 0 & 5 & 5 \\
\hline$J_{2}$ & 1 & 4 & 6 \\
\hline$J_{3}$ & 3 & 1 & 8 \\
\hline
\end{tabular}

Table 1: An Instance of $1\left|r_{i}\right| \sum T_{i}$.

Proposition 1. Let $[i]$ denote the index of the job which is completed in the 
$i^{\text {th }}$ position in the SRPT schedule. The total tardiness is at least

$$
\sum_{i=1}^{n} \max \left(C_{[i]}-d_{i}, 0\right) .
$$

\subsection{A New Lower Bound}

We introduce two propositions, that are valid in the preemptive case only. Proposition 2 is a weak version of the Generalized Emmons Rule 1. Proposition 3 shows that under some conditions, due dates can be exchanged without increasing the objective function.

Proposition 2. Let $J_{i}$ and $J_{k}$ be two jobs such that $r_{i} \leq r_{k}, p_{i} \leq p_{k}$ and $d_{i} \leq d_{k}$, then there exists an optimal schedule in which $J_{k}$ starts after the end of $J_{i}$.

Proof. See proof of Generalized Emmons Rule 1.

Proposition 3. Let $J_{i}$ and $J_{k}$ be two jobs such that $r_{i} \leq r_{k}, p_{i} \leq p_{k}$ and $d_{i}>d_{k}$. Exchanging $d_{i}$ and $d_{k}$ does not increase the optimal total tardiness.

Proof. Consider an optimal schedule $S$ of the original instance and let $C_{i}$ and $C_{k}$ be the completion times of $J_{i}$ and $J_{k}$ in $S$. Let $\Delta$ be the difference between the tardiness of $S$ before and after the exchange.

$\Delta=\max \left(0, C_{i}-d_{k}\right)+\max \left(0, C_{k}-d_{i}\right)-\max \left(0, C_{i}-d_{i}\right)-\max \left(0, C_{k}-d_{k}\right)$.

First, assume that $C_{i}<C_{k}$. Note that $\Delta=\max \left(d_{k}, C_{i}\right)+\max \left(d_{i}, C_{k}\right)-$ $\max \left(d_{i}, C_{i}\right)-\max \left(d_{k}, C_{k}\right)$. If $d_{i}>C_{k}$ then $d_{i}>C_{i}$ and $\max \left(d_{i}, C_{k}\right)-$ $\max \left(d_{i}, C_{i}\right)=0$. Moreover, $\max \left(d_{k}, C_{i}\right)-\max \left(d_{k}, C_{k}\right) \leq 0$ since $C_{i}<C_{k}$. This leads to $\Delta \leq 0$. Now, if $d_{i} \leq C_{k}$ then $d_{k}<C_{k}$ since $d_{i}>d_{k}$ and 
$\max \left(d_{i}, C_{k}\right)-\max \left(d_{k}, C_{k}\right)=0$. Moreover, $\max \left(d_{k}, C_{i}\right)-\max \left(d_{i}, C_{i}\right) \leq 0$ since $d_{i}>d_{k}$. This leads to $\Delta \leq 0$.

Now assume that $C_{k}<C_{i}$. After the exchange of due dates, we exchange the pieces of the jobs, such that all pieces of $J_{i}$ are scheduled before those of $J_{k}$. This exchange is valid since $r_{i} \leq r_{k}$. In this new schedule, $J_{i}$ is completed before or at $C_{k}$ because $p_{i} \leq p_{k}$, thus the tardiness of $J_{i}$ is lower than or equal to $\max \left(0, C_{k}-d_{k}\right)$. Moreover, $J_{k}$ is completed at $C_{i}$ and its tardiness is equal to $\max \left(0, C_{i}-d_{i}\right)$. Thus $\Delta \leq \max \left(0, C_{i}-d_{i}\right)+\max \left(0, C_{k}-d_{k}\right)-\max \left(0, C_{i}-\right.$ $\left.d_{i}\right)-\max \left(0, C_{k}-d_{k}\right) \leq 0$.

These propositions allow us to compute a lower bound $\boldsymbol{l} \boldsymbol{b}_{\mathbf{1}}$ thanks to the following algorithm.

At each time $t$, we consider $D=\left\{J_{j} / r_{j} \leq t \wedge p_{j}^{\prime}>0\right\}$ the set of jobs available but not completed at $t\left(p_{j}^{\prime}\right.$ denotes the remaining processing time of $J_{j}$ at time $t$ ). Let $J_{u}$ be the job with the shortest remaining processing time, and let $J_{v}$ be the job with the smallest due date. If $d_{u}=d_{v}$, i.e., $J_{u}$ has the smallest due date, according to Proposition 2, it is optimal to schedule one unit of this job. If it is not the case, according to Proposition 3, we exchange its due date with the one of $J_{v}$, the job with the smallest due date. This new instance has an optimal total tardiness lower than or equal to the optimal tardiness of the original problem. In this new problem, $J_{u}$ has now the smallest due date and the smallest remaining processing time, then it is optimal to schedule one unit of this job according to Proposition 2. We increase $t$ and we iterate until all jobs are completed.

At each time $t$, we build a new problem by exchanging two due dates. This new problem has an optimal total tardiness lower than or equal to the 
problem before. Hence, at the end of the algorithm, we obtain an optimal schedule of a problem which has a total tardiness lower than or equal to the optimal tardiness of the original problem. Therefore, it is a lower bound of the original problem. Actually, the only relevant time points are when a job becomes available or when a job is completed. And so there are at most $2 n$ times $t$ to consider.

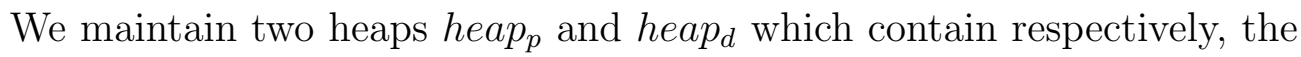
uncompleted jobs which are available at time $t$ sorted in non-decreasing order of remaining processing times, and these jobs sorted in non-decreasing order of due dates. The insertion, the extraction and the modification of a job of one heap costs $O(\log n)$. Getting the minimum element of one heap costs $O(1)$. We have at most $n$ insertions (each time a job becomes available), and at most $n$ extractions (each time a job is completed). At each time $t$, we have at most two modifications in heap $p$ and in heap $p_{d}$ when we must exchange the due dates of two jobs. Hence, our algorithm runs in $O(n \log n)$.

\subsection{Comparison with Chu's Lower Bound}

The schedule built by our algorithm is the same as the one built by Chu. The algorithms differ from the assignment of the due dates to the jobs. Indeed, in the algorithm of $\mathrm{Chu}$, the assignment of the due dates is performed at a "global" level, whereas in our algorithm, the assignment is performed at a "local" level. We show that $l b_{1}$ is strictly better than $l b_{C h u}$.

Proposition 4. The lower bound $l b_{1}$ obtained by our algorithm is stronger than Chu's lower bound $l b_{C h u}$. 
Proof. The sets of completion times in the schedules built by our algorithm and by Chu's are the same. The difference lies in the assignment of due dates to the jobs. Let $\left(d_{1}, \ldots, d_{n}\right)$ be the sequence of due dates sorted in non-decreasing order. This is the sequence obtained by the algorithm of Chu. Let $\left(d_{\sigma(1)}, \ldots, d_{\sigma(n)}\right)$ be the sequence of due dates obtained by our algorithm. Let $j$ be the first index such that $j \neq \sigma(j)$ and let $t$ be the starting time of the piece of job which is completed at $C_{[j]}$. This piece is a piece of the job with the shortest remaining processing time at $t$. We have $d_{j} \in\left(d_{\sigma(j+1)}, \ldots, d_{\sigma(n)}\right)$. Let $k$ be the integer such that $\sigma(k)=j$. Note that $d_{\sigma(k)} \leq d_{\sigma(j)}$. Suppose we exchange the due dates $d_{\sigma(j)}$ and $d_{\sigma(k)}$ in the sequence $\left(d_{\sigma(j)}, \ldots, d_{\sigma(n)}\right)$. As a result, the decrease of the total tardiness is equal to $\max \left(0, C_{[i]}-d_{\sigma(j)}\right)+\max \left(0, C_{[k]}-d_{\sigma(k)}\right)-\max \left(0, C_{[i]}-d_{\sigma(k)}\right)-$ $\max \left(0, C_{[k]}-d_{\sigma(j)}\right)$ which is non-negative as shown in the first part of the Proposition 3 because $C_{[i]}<C_{[k]}$ and $d_{\sigma(k)} \leq d_{\sigma(j)}$. Now the two sequences are identical up to index $j+1$. We iterate until the sequences are the same. Hence $l b_{C h u} \leq l b_{1}$.

We provide an example for an instance with 3 jobs, for which $l b_{1}$ is equal to 1 whereas $l b_{C h u}$ is equal to 0 (see Table 2 and Figure 6). For this instance, $l b_{1}$ is the optimal total tardiness.

\subsection{Improving the Lower Bound}

To improve our lower bound, we extensively use the Generalized Emmons Rules as a pre-processing step before the computation of the lower bound. The lower bound is valid in the preemptive case hence, we can apply the 


\begin{tabular}{|c|c|c|c|}
\hline$J_{i}$ & $r_{i}$ & $p_{i}$ & $d_{i}$ \\
\hline \hline$J_{1}$ & 0 & 1 & 3 \\
\hline$J_{2}$ & 1 & 1 & 2 \\
\hline$J_{3}$ & 1 & 1 & 2 \\
\hline
\end{tabular}

Table 2: Example with $l b_{1}>l b_{C h u}$.

Generalized Emmons Rules. They allow us to tighten the release dates, which has a dramatic impact on the value of the lower bound.

Recall that, to compute our lower bound, we chronologically build a preemptive schedule (see Section 3.2). We still follow the same algorithm but, at each time point where a piece of job to schedule next has to be chosen, we apply the Generalized Emmons Rules on the jobs that are not scheduled yet.

Since there are $O(n)$ relevant time points and since the propagation of the Generalized Emmons Rules runs in $O\left(n^{3}\right)$, the improved lower bound can be computed in $O\left(n^{4}\right)$. In practice, the propagation of the Generalized Emmons Rules is much "faster" than $O\left(n^{3}\right)$ and the bound is computed in a reasonable amount of time. In the following we use the notation $\boldsymbol{l} \boldsymbol{b}_{\mathbf{2}}$ to refer to this bound. 


\section{Propagation Rules}

\subsection{Possible and Impossible Positions}

Focacci [11] has recently proposed an original approach based on Constraint Programming to compute a lower bound of $1\left|r_{i}\right| \sum T_{i}$. In this approach, each job is associated with a constrained variable identifying all possible positions (first, second, third, etc.) that the job can assume in a schedule. Following this idea, we present some rules that deduce that a job cannot be executed in some positions. This information allows us to adjust the release dates and to "filter" the sets $P F$ and $P L$.

From now on, we assume that jobs are sorted in non-decreasing order of due dates. To simplify the presentation, we also assume that no job has been sequenced in the right part of the schedule, i.e., $P=\emptyset$. If $P$ is not empty, we can "remove" the jobs of $P$ and apply the rules described below. Of course, the tardiness of the jobs that were in $P$ has to be added to the lower-bounds computed below.

As in Section 3.1, [i] denotes the index of the job which is completed in the $i^{\text {th }}$ position in the SRPT schedule. We know that $C_{[i]}$ is a lower bound of the completion time of the job scheduled in $i^{\text {th }}$ position and according to Proposition 1, $\sum_{i=1}^{n} \max \left(C_{[i]}-d_{i}, 0\right)$ is a lower bound of the total tardiness (each job $J_{i}$ is assigned to the completion time $C_{[i]}$ ).

Suppose now that we want to compute a lower bound of the total tardiness under the hypothesis that $J_{i}$ is scheduled in the $k^{\text {th }}$ position. We first assign the completion time $C_{[k]}$ to $J_{i}$ and we reassign all other completion times to all other jobs as follows. 
- If $k<i$ the jobs $J_{k}, J_{k+1}, \ldots, J_{i-1}$ are assigned to $C_{[k+1]}, C_{[k+2]}, \ldots, C_{[i]}$ and the other assignments do not change.

- If $k>i$ the jobs $J_{i+1}, J_{i+2}, \ldots, J_{k}$ are assigned to $C_{[i]}, C_{[i+1]}, \ldots, C_{[k-1]}$ and the other assignments do not change.

Following these new assignments, we have a new lower bound. If it is greater than $\bar{T}$ (the upper bound of the objective function, as defined in Section 1), then $J_{i}$ cannot be in the $k^{t h}$ position.

Now assume that we have shown that positions $1, \ldots, k$ are not possible for a job $J_{i}$ then $J_{i}$ cannot be completed before $C_{[k+1]}$ and cannot start before $C_{[k]}$. Hence, the release date $r_{i}$ can be adjusted to $\max \left(r_{i}, C_{[k+1]}-p_{i}, C_{[k]}\right)$. Moreover, if $k>1$ then $i$ cannot be scheduled first, i.e., $J_{i}$ can be removed of $P F$. Of course, symmetric rules hold when it is known that the last $k$ positions are not possible for $J_{i}$.

To implement this constraint propagation rule, we just have to use the $O(n \log n)$ algorithm of $\mathrm{Chu}[8]$, to compute the values $C_{[1]}, C_{[2]} \ldots, C_{[n]}$. Then, for each job $J_{i}$ and for each position $k$, the lower-bound can be recomputed in linear time thanks to the reassignment rules provided above. This leads to an overall time complexity of $O\left(n^{3}\right)$.

Actually, this algorithm can be improved as follows. Assume that we have computed the lower bound under the assumption that $J_{i}$ is scheduled in position $k$. To compute the lower bound under the assumption that $J_{i}$ is scheduled in position $k-1$, we just have to exchange the assignments of job $i$ and job $[k-1]$. The modification of the lower bound is then $\max \left(0, C_{[k-1]}-\right.$ $\left.d_{i}\right)-\max \left(0, C_{[k]}-d_{i}\right)+\max \left(0, C_{[k]}-d_{[k-1]}\right)-\max \left(0, C_{[k-1]}-d_{[k-1]}\right)$. Hence, we can "try" all possible positions for $J_{i}$ in linear time. All impossible positions 
can thus be computed in $O\left(n^{2}\right)$.

\subsection{Look-Ahead}

We use a kind of look-ahead technique to test whether a job $J_{i}$ can be removed of the set of possible first: The job $J_{i}$ is sequenced immediately after $P$ and a lower bound of the new scheduling situation $\left(l b_{2}\right.$ in the current implementation) is computed. If this lower bound is greater than $\bar{T}$ then $J_{i}$ cannot be first and it is removed from $P F$. A symmetric rule is used for the $P L$ set.

\section{$5 \quad$ Experimental Results}

All techniques presented in this paper have been incorporated into a Branchand-Bound method implemented on top of Ilog Solver and Ilog SchedULER. All experimental results have been computed on a PC Dell Latitude $650 \mathrm{MHz}$ running Windows 98.

The instances have been generated with the scheme of Chu [8]. Each instance is generated randomly from three uniform distributions of $r_{i}, p_{i}$ and $d_{i}$. The distribution of $p_{i}$ is always between 1 and 10 . The distributions of $r_{i}$ and $d_{i}$ depend on 2 parameters: $\alpha$ and $\beta$. For each job $J_{i}, r_{i}$ is generated from the distribution $\left[0, \alpha \sum p_{i}\right]$ and $d_{i}-\left(r_{i}+p_{i}\right)$ is generated from the distribution $\left[0, \beta \sum p_{i}\right]$. Four values for $\alpha$ and three values for $\beta$ were combined to produce 12 instances sets, each containing 10 instances of $n$ jobs with $n \in\{10,20,30,40,50, \ldots, 500\}$ jobs.

In Table 3, Chu's lower bound is compared with the new lower bound $l b_{2}$ 


\begin{tabular}{||r||r|r|r|r||r|r|r|r||}
\hline \multicolumn{1}{||c||}{} & \multicolumn{4}{|c||}{ Chu's Lower Bound } & \multicolumn{4}{|||}{$l b_{2}$} \\
\hline Opt. & $l b_{\text {Chu }}$ & Gap & Bck. & CPU & $l b_{2}$ & Gap & Bck. & CPU \\
34 & 28 & 0.21 & 501340 & 773.9 & 34 & 0.00 & 0 & 0.82 \\
111 & 46 & 1.41 & - & - & 111 & 0.00 & 0 & 0.06 \\
115 & 35 & 2.29 & - & - & 93 & 0.24 & 786 & 0.61 \\
95 & 47 & 1.02 & 603171 & 1313.33 & 81 & 0.17 & 10361 & 7.47 \\
32 & 19 & 0.68 & 24913 & 56.63 & 31 & 0.03 & 172 & 0.17 \\
12 & 9 & 0.33 & 1 & 0.06 & 12 & 0.00 & 0 & 0 \\
79 & 40 & 0.98 & - & - & 67 & 0.18 & 283 & 0.33 \\
192 & 167 & 0.15 & 10118 & 28.18 & 180 & 0.07 & 790 & 0.6 \\
84 & 54 & 0.56 & 52922 & 79.8 & 79 & 0.06 & 14 & 0.05 \\
24 & 16 & 0.50 & 69754 & 89.36 & 24 & 0.00 & 0 & 0 \\
\hline
\end{tabular}

Table 3: Comparison Between $l b_{C h u}$ and $l b_{2} . n=20$ Jobs, $\alpha=0.5, \beta=0.5$, no Dominance Property, no Intelligent Backtracking. 
(see Section 3.4). Most times, $l b_{1}=l b_{C h u}$ so, $l b_{1}$ has been skipped from the tables to simplify the presentation. To have a fair comparison of the bounds, the Branch-and-Bound procedure has been run without dominance property nor Intelligent Backtracking on instances with 20 jobs that are known to be hard $(\alpha=0.5, \beta=0.5)[8]$. Each line corresponds to a single instance and we report the optimal tardiness (Opt.), the value of the lower bound computed at the root of search tree $\left(l b_{C h u}, l b_{2}\right)$, the relative gap (Gap) between the optimum and the two lower bounds, the number of backtracks (Bck.) and the amount of CPU time in seconds required to solve the instance. A "_" in the table indicates that the search was interrupted after 1800 seconds. On the average, $l b_{C h u}$ is at $81 \%$ of the optimum value while our lower bound is much closer $(6 \%)$.

In Table 4, we show the efficiency of dominance properties, propagation rules and intelligent backtracking technique presented in this paper. For that, the Branch-and-Bound procedure has been run with $l b_{2}$ on instances with 30 jobs with various combinations of $\alpha$ and $\beta$. On each line of Table 4 , the average results obtained over the 10 generated instances are reported. In columns 3 and 4 ("Chu"), we report the results obtained when the dominance properties of Chu are used $[7,8]$. We then add (columns 5 and 6) the dominance and propagation rules presented in Section 2.1.4 (EQual Processing time, EQP), Section 2.2 (Dominance rules relying on Scheduled Jobs, DSJ) and Section 4.1 (Possible and Impossible Positions, PIP). Intelligent Backtracking (IB) is then added and results are reported in columns 7 and 8. Finally, the results obtained with the look-ahead (LAH) technique are described in columns 9 and 10 . 


\begin{tabular}{|c|c|c|c|c|c|c|c|c|c|}
\hline & & \multicolumn{2}{|c|}{ Chu } & \multicolumn{2}{|c|}{$\begin{array}{l}\text { Chu, EQP } \\
\text { DSJ, PIP }\end{array}$} & \multicolumn{2}{|c|}{$\begin{array}{c}\text { Chu, EQP } \\
\text { DSJ, PIP } \\
\text { IB }\end{array}$} & \multicolumn{2}{|c|}{$\begin{array}{c}\text { Chu, EQP } \\
\text { DSJ, PIP } \\
\text { IB, LAH }\end{array}$} \\
\hline$\alpha$ & $\beta$ & Bck. & $\mathrm{CPU}$ & Bck. & $\mathrm{CPU}$ & Bck. & $\mathrm{CPU}$ & Bck. & $\mathrm{CPU}$ \\
\hline 0 & 0.05 & 0 & 0.03 & 0 & 0.03 & 0 & 0.05 & 0 & 0.02 \\
\hline 0 & 0.25 & 6 & 0.05 & 5 & 0.04 & 5 & 0.04 & 5 & 0.08 \\
\hline 0 & 0.5 & 64 & 0.10 & 62 & 0.12 & 62 & 0.13 & 62 & 0.21 \\
\hline 0.5 & 0.05 & 91 & 0.19 & 23 & 0.13 & 20 & 0.13 & 5 & 0.27 \\
\hline 0.5 & 0.25 & 3990 & 6.55 & 188 & 1.18 & 172 & 1.11 & 58 & 1.67 \\
\hline 0.5 & 0.5 & 8468 & 12.25 & 349 & 2.05 & 297 & 1.76 & 107 & 2.14 \\
\hline 1 & 0.05 & 188 & 0.28 & 29 & 0.13 & 23 & 0.12 & 9 & 0.24 \\
\hline 1 & 0.25 & 698 & 0.85 & 47 & 0.24 & 30 & 0.16 & 12 & 0.21 \\
\hline 1 & 0.5 & 0 & 0.02 & 0 & 0.01 & 0 & 0.01 & 0 & 0.02 \\
\hline 1.5 & 0.05 & 16 & 0.03 & 7 & 0.05 & 6 & 0.04 & 3 & 0.04 \\
\hline 1.5 & 0.25 & 18 & 0.04 & 9 & 0.03 & 7 & 0.05 & 5 & 0.06 \\
\hline 1.5 & 0.5 & 1 & 0.02 & 1 & 0.01 & 1 & 0.01 & 1 & 0.03 \\
\hline
\end{tabular}

Table 4: Efficiency of Dominance Properties, Propagation Rules and Intelligent Backtracking. 
All "ingredients" described in this paper are useful to reduce the search space. However, the look ahead technique presented in Section 4.2 seems to be very costly in terms of CPU time compared to the corresponding reduction of the search tree. This is due to the relatively high complexity of the lower bound $l b_{2}$ that is used several times in the look ahead. We tried to use some weaker lower bound like $l b_{1}$ but it does not reduce the search space.

The results obtained with the version of the algorithm that incorporates all ingredients except the look-ahead technique are presented in Table 5. For each combination of parameters and for each value of $n$, we provide the average number of fails and the average computation time in seconds. A time limit of 3600 seconds has been fixed. All instances are solved within the time limit for up to 50 jobs. For $n=60$, and for $(\alpha=0.5, \beta=0.5)$, most of the instances cannot be solved. As noticed earlier by Chu [8], instances generated according to this particular combination seem to be "hard" to solve in practice.

From this table, we can remark that the "hardness" increases very quickly with $n$, especially for $(\alpha=0.5, \beta=0.5)$. For Each combination of parameters, we report the largest size of instance (column "Largest") for which $80 \%$ of instances are solved within one hour of CPU time. In practice most of the instances are solved within 30 seconds and our results compare well to those of [8]. For instance, the average number of fails for the combination $(\alpha=0.5, \beta=0.5)$ was greater than 36000 in [8], whereas this number is now lower than 300 . 


\begin{tabular}{|c|c|c|c|c|c|c|c|c|c|c|c|c|c|c|}
\hline \multirow[b]{2}{*}{$\alpha$} & \multirow[b]{2}{*}{$\beta$} & \multicolumn{2}{|c|}{$n=10$} & \multicolumn{2}{|c|}{$n=20$} & \multicolumn{2}{|c|}{$n=30$} & \multicolumn{2}{|c|}{$n=40$} & \multicolumn{2}{|c|}{$n=50$} & \multicolumn{2}{|c|}{$n=60$} & \multirow[t]{2}{*}{ Largest } \\
\hline & & Bck. & $\mathrm{CPU}$ & Bck. & $\mathrm{CPU}$ & Bck. & $\mathrm{CPU}$ & Bck. & $\mathrm{CPU}$ & Bck. & $\mathrm{CPU}$ & Bck. & $\mathrm{CPU}$ & \\
\hline 0 & 0.05 & 0 & 0.06 & 0 & 0.01 & 0 & 0.01 & 1 & 0.04 & 2 & 0.09 & 0.00 & 0.08 & 300 \\
\hline 0 & 0.25 & 0 & 0.02 & 2 & 0.02 & 5 & 0.03 & 17 & 0.11 & 14 & 0.21 & 38.90 & 0.46 & 120 \\
\hline 0 & 0.5 & 1 & 0.00 & 6 & 0.02 & 62 & 0.11 & 52 & 0.25 & 218 & 0.95 & 1205.00 & 8.62 & 80 \\
\hline 0.5 & 0.05 & 2 & 0.02 & 14 & 0.05 & 20 & 0.12 & 71 & 0.66 & 104 & 1.33 & 335.70 & 7.20 & 90 \\
\hline 0.5 & 0.25 & 3 & 0.02 & 30 & 0.12 & 172 & 1.10 & 621 & 6.88 & 2516 & 45.48 & 8708.50 & 227.40 & 60 \\
\hline 0.5 & 0.5 & 3 & 0.02 & 39 & 0.14 & 297 & 1.76 & 2824 & 31.97 & 12572 & 203.12 & & - & 50 \\
\hline 1 & 0.05 & 1 & 0.02 & 10 & 0.04 & 23 & 0.13 & 49 & 0.37 & 95 & 1.30 & 122.80 & 2.90 & 90 \\
\hline 1 & 0.25 & 1 & 0.01 & 12 & 0.04 & 30 & 0.17 & 82 & 0.70 & 106 & 1.27 & 266.00 & 3.70 & 500 \\
\hline 1 & 0.5 & 1 & 0.01 & 23 & 0.08 & 0 & 0.01 & 10 & 0.07 & 4 & 0.06 & 0.00 & 0.05 & 500 \\
\hline 1.5 & 0.05 & 1 & 0.03 & 5 & 0.02 & 6 & 0.04 & 29 & 0.24 & 30 & 0.34 & 36.20 & 0.66 & 190 \\
\hline 1.5 & 0.25 & 0 & 0.00 & 2 & 0.01 & 7 & 0.03 & 0 & 0.02 & 19 & 0.12 & 6.40 & 0.10 & 500 \\
\hline 1.5 & 0.5 & 0 & 0.01 & 0 & 0.02 & 1 & 0.01 & 0 & 0.03 & 0 & 0.04 & 0.00 & 0.05 & 500 \\
\hline
\end{tabular}

Table 5: Results Obtained with up to 60 Jobs. 


\section{Conclusion}

We have presented new lower-bounds, new constraint propagation techniques and new dominance properties for $1\left|r_{i}\right| \sum T_{i}$. Computational results show that the proposed approach outperforms the best known procedures. We think that several techniques presented in this paper can be extended to more complex criteria such as weighted tardiness or weighted flow time.

\section{Acknowledgments}

The authors would like to thank Chengbin Chu, Federico Della Croce, Filippo Focacci and Andrea Grosso for enlightening discussions on total tardiness. The authors are also grateful to anonymous referees for their suggestions regarding the improvement of this article.

\section{References}

[1] T.S. Abdul-Razacq, C.N. Potts and L.N. Van Wassenhove, A survey of algorithms for the single machine total weighted tardiness scheduling problem, Discrete Applied Mathematics 26 (1990)235-253.

[2] Ph. Baptiste, C. Le Pape and W. Nuijten, Constraint-Based Scheduling, Applying Constraint Programming to Scheduling Problems, International Series In Operations Research And Management Science Volume 39, Kluwer, 2001. 
[3] Ph. Baptiste and A. Jouglet, On minimizing total tardiness in a serial batching problem, RAIRO Operational Research 35 (2001)107-115.

[4] J. Carlier, Ordonnancements à contraintes disjonctives, RAIRO 12 (1978)333-351.

[5] J. Carlier and E. Pinson, A Practical Use of Jackson's Preemptive Schedule for Solving the Job-Shop Problem, Annals of Operations Research 26 (1990)269-287.

[6] S. Chang, Q. Lu, G. Tang and W. Yu, On decomposition of the total tardiness problem, Operations Research 17 (1995)221-229.

[7] C. Chu and M.-C. Portmann, Some new efficient methods to solve the $n|1| r_{i} \mid \sum T_{i}$ scheduling problem, European Journal of Operational Research 58 (1991)404-413.

[8] C. Chu, A Branch-and-Bound algorithm to minimize total tardiness with different release dates, Naval Research Logistic 39 (1992)265-283.

[9] J. Du, J.Y.T. Leung, Minimizing total tardiness on one processor is NP-Hard, Mathematics of Operations Research 15 (1990)483-495.

[10] H. Emmons, One-machine sequencing to minimize certain functions of job tardiness, Operations Research 17 (1969)701-715.

[11] F. Focacci, Solving Combinatorial Optimization Problems in Constraint Programming, PhD Thesis, University of Ferrara, Italy, 2000, 101-104.

[12] E.L. Lawler, A pseudo-polynomial algorithm for sequencing jobs to minimize total tardiness, Annals of Discrete Mathematics 1 (1977)331-342. 
[13] O. Lhomme, Consistency Techniques for Numeric CSPs, Proceedings, 13th International Joint Conference on Artificial Intelligence, 1993.

[14] C.N. Potts, L.N. Van Wassenhove, A decomposition algorithm for the single machine total tardiness problem, Operations Research Letters 26 (1982)177-182.

[15] A.H.G. Rinnooy Kan, Machine sequencing problem: classification, complexity and computation, Nijhoff, The Hague, 1976.

[16] W. Szwarc, F. Della Croce and Grosso, Solution of the single-machine total tardiness problem, Journal of Scheduling 2 (1999)55-71.

[17] W. Szwarc, A. Grosso and F. Della Croce, Algorithmic Paradoxes of the single-machine total tardiness problem, Journal of Scheduling 4 (2001)93-104. 


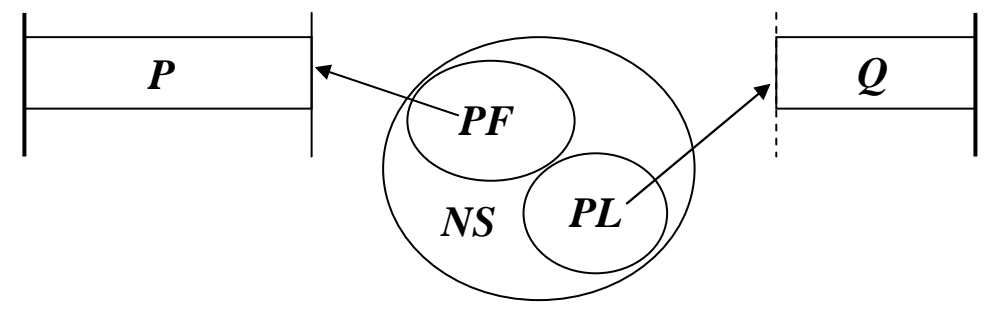

Figure 1: A Node of the Search Tree. 


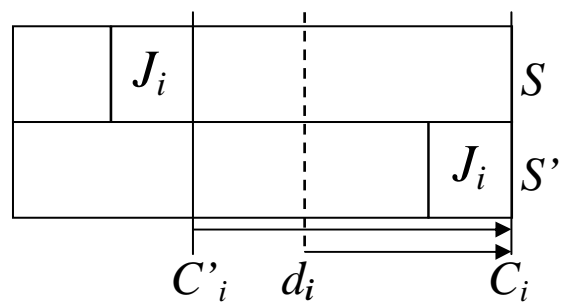

Figure 2: Case where $C_{i}^{\prime}<C_{i}$ and $d_{i} \leq C_{i}$. 


\begin{tabular}{|c|c|c|c|c|}
\hline$J_{k}$ & $J_{k}$ & $J_{i}$ & $J_{k}$ & $J_{i}$ \\
\hline & & & & \\
\hline$J_{i}$ & $J_{i}$ & $J_{k}$ & $J_{k}$ & $J_{k}$ \\
\hline
\end{tabular}

Figure 3: Generalized Emmons Rules. 


\begin{tabular}{|c|c|c|c|c|}
\hline \multicolumn{2}{|c|}{$J_{2}$} & $J_{3}$ & $J_{1}$ & $J_{4}$ \\
\hline$J_{1}$ & $J_{2}$ & $J_{3}$ & $J_{2}$ & $J_{4}$ \\
\hline
\end{tabular}

Figure 4: An Optimal Non-Preemptive Schedule and an Optimal Preemptive Schedule. 


\begin{tabular}{|l|l|l|l|l|l|l|}
\hline \multicolumn{1}{c}{$d_{1}$} & $d_{2}$ & $d_{3}$ \\
\hline$J_{1}$ & $J_{2}$ & $J_{3}$ & $J_{2}$ & & $J_{1}$ \\
\hline & \multicolumn{1}{c}{$C_{[1]}$} & $C_{[2]}$ & $C_{[3]}$
\end{tabular}

Figure 5: An SRPT Schedule of the Instance of Table 1. 


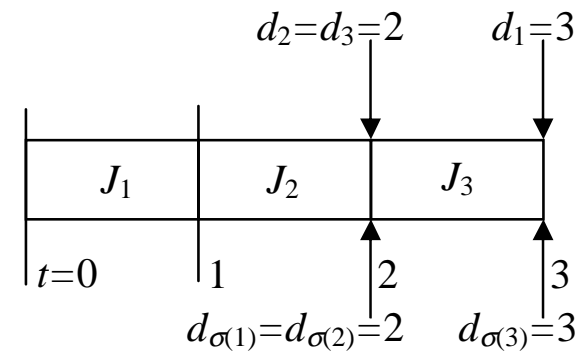

Figure 6: Assignment of Due Dates Obtained by the Algorithm Computing $l b_{1}\left(d_{1}, d_{2}, d_{3}\right)$ and by Chu's Algorithm $\left(d_{\sigma(1)}, d_{\sigma(2)}, d_{\sigma(3)}\right)$. 